\title{
Lower bounds for the number of integral polynomials with given order of discriminants
}

by

\author{
Vasili Bernik (Minsk), Friedrich Götze (Bielefeld) \\ and Olga KuKso (Minsk)
}

\section{Dedicated to Professor Wolfgang Schmidt}

1. Introduction. The discriminant of a polynomial is a vital characteristic that crops up in various problems of number theory. For example, they play an important role in Diophantine equations, Diophantine approximation and algebraic number theory [3-7].

Let

$$
P(x)=a_{n} x^{n}+a_{n-1} x^{n-1}+\cdots+a_{1} x+a_{0}=a_{n}\left(x-\alpha_{1}\right) \cdots\left(x-\alpha_{n}\right)
$$

be a polynomial. By definition,

$$
D(P)=a_{n}^{2 n-2} \prod_{1 \leq i<j \leq n}\left(\alpha_{i}-\alpha_{j}\right)^{2} .
$$

It is also well known that

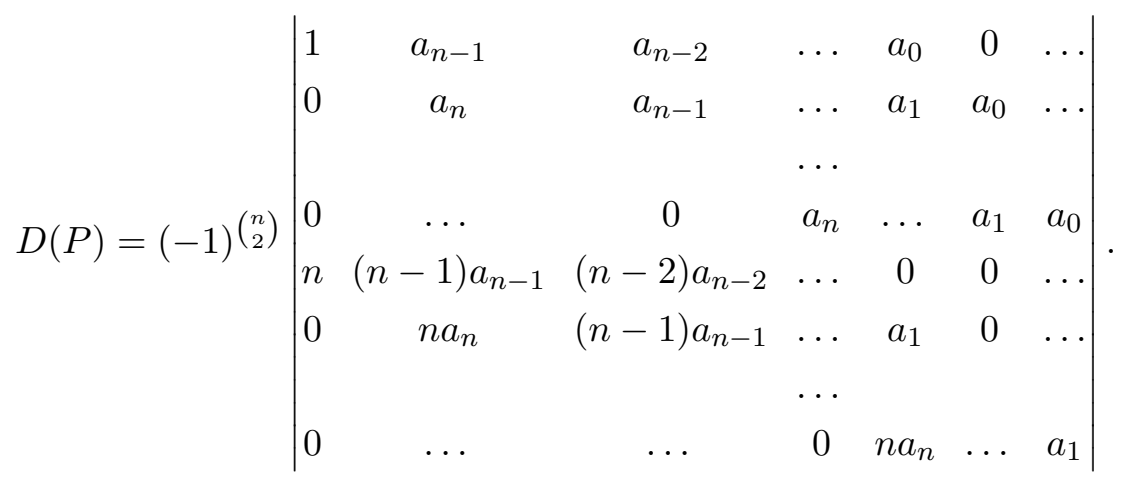

2000 Mathematics Subject Classification: 11J83, 11J20.

Key words and phrases: Diophantine approximations, discriminant, Minkowski's theorem on linear forms. 
Trivially, by (2), whenever $P$ has rational integral coefficients the discriminant $D(P)$ is also an integer. Furthermore, if $D(P) \neq 0$ then

$$
|D(P)| \geq 1 \text {. }
$$

Also (1) obviously implies that $D(P) \neq 0$ if and only if $P(x)$ has no multiple roots.

Fix $n \in \mathbb{N}$. Let $Q>Q_{0}(n)$, where $Q_{0}(n)$ is a sufficiently large number. Throughout $\mathcal{P}_{n}(Q)$ will denote the class of non-zero polynomials $P(x)$ with $\operatorname{deg} P \leq n$ and $H(P) \leq Q$. Furthermore, $\mathcal{P}_{n}^{\prime}(H)$ will be the subclass of $\mathcal{P}_{n}(H)$ consisting of polynomials $P$ with $H(P)=H$. In what follows $c(n)$ and $c_{j}, j=0,1, \ldots$, will stand for some positive constants depending on $n$ only. Also we will use the Vinogradov symbol $A \ll B$ meaning that $A \leq c_{0} B$. The notation $A \asymp B$ means $B \ll A \ll B$.

Using the representation (2) for $D(P)$ one readily verifies that $|D(P)|<$ $c(n) Q^{2 n-2}$. Thus, by (3), we have

$$
1 \leq|D(P)|<c(n) Q^{2 n-2}
$$

for integral polynomials $P$ with no multiple roots.

The number of polynomials in the class $\mathcal{P}_{n}(Q)$ is finite and is easily verified to satisfy

$$
\# \mathcal{P}_{n}(Q)<2^{2 n+2} Q^{n+1} .
$$

The latter together with (4) shows that $\left[1, c(n) Q^{2 n-2}\right]$ contains intervals of length $c(n) Q^{n-3}$ free from values of discriminants of $P \in \mathcal{P}_{n}(Q)$. For $n \geq 4$ these intervals can be arbitrarily large. Thus, the discriminants $D(P)$ are rather sparse in the interval $\left[1, c(n) Q^{2 n-2}\right]$ they belong to. In this paper we establish a sharp lower bound for the number of polynomials $P \in \mathcal{P}_{n}(Q)$ with relatively small discriminants. To the best of our knowledge this is the first result of this kind.

2. Main theorems. In this paper we prove two theorems. The first one deals with the distribution of discriminants of integral polynomials.

Theorem 1. Let $v \in[0,1 / 2]$. Then there are at least $c(n) Q^{n+1-2 v}$ polynomials $P$ in $\mathcal{P}_{n}(Q)$ with

$$
|D(P)|<Q^{2 n-2-2 v} .
$$

In all likelihood the lower bound on the number of polynomials satisfying (5) is best possible up to a constant multiple. Establishing this would also give a lower bound in (5).

The following is an effective metrical result that represents the key to establishing Theorem 1 . Let $Q$ denote a sufficiently large number, $v \in[0,1 / 2]$ and let $c_{1}$ and $c_{2}$ be positive constants. By Minkowski's theorem on linear 
forms, for any $x \in[-1 / 2,1 / 2]$ the system of inequalities

$$
\left\{\begin{array}{l}
|P(x)|<c_{1} Q^{-n+v} \\
\left|P^{\prime}(x)\right|<c_{2} Q^{1-v}
\end{array}\right.
$$

has a solution in polynomials $P \in \mathcal{P}_{n}(Q)$ whenever $c_{1} c_{2}>1$. Our next result shows that the condition $c_{1} c_{2}>1$ cannot be substantially relaxed. To state the result we introduce further notation. Let $\mathcal{L}_{n, Q}\left(c_{1}, c_{2}\right)$ denote the set of $x \in I \subset[-1 / 2,1 / 2]$ such that (6) has a solution in $P \in \mathcal{P}_{n}(Q)$.

TheOREM 2. Let $Q$ denote a sufficiently large number, $v \in[0,1 / 2]$ and let $c_{1}$ and $c_{2}$ be positive constants such that $c_{1} c_{2}<n^{-1} 2^{-n-11}$. Then

$$
\mu \mathcal{L}_{n, Q}\left(c_{1}, c_{2}\right)<|I| / 2,
$$

where $\mu$ denotes the Lebesgue measure on the real axis.

3. Auxiliary lemmas. This section contains several lemmas that will be used in the course of establishing Theorem 2 .

For each polynomial $P(x) \in \mathbb{Z}[x]$ of degree $n$ with roots $\alpha_{1}, \ldots, \alpha_{n}$, that is,

$$
P(x)=a_{n}\left(x-\alpha_{1}\right) \cdots\left(x-\alpha_{n}\right),
$$

we pick a root, say $\alpha_{1}$, and consider only those $x \in I$ with $\min _{1 \leq i \leq n}\left|x-\alpha_{i}\right|=$ $\left|x-\alpha_{1}\right|$. Furthermore, order the other roots of $P$ according to the distance from $\alpha_{1}$ so that

$$
\left|\alpha_{1}-\alpha_{2}\right| \leq\left|\alpha_{1}-\alpha_{3}\right| \leq \cdots \leq\left|\alpha_{1}-\alpha_{n}\right| .
$$

Define vectors $\left(\mu_{2}, \mu_{3}, \ldots, \mu_{n}\right)$ and $\left(l_{2}, l_{3}, \ldots, l_{n}\right)$ by setting

$$
\left|\alpha_{1}-\alpha_{j}\right|=H^{-\mu_{j}}, \quad l_{j}-1=\left[\mu_{j} T\right], \quad j=\overline{2, n},
$$

where $T=[n / \varepsilon]+1$ and $\varepsilon$ is a small positive number. It is readily seen that $\left(l_{j}-1\right) T^{-1} \leq \mu_{j}<l_{j} T^{-1}$. Further, define

$$
p_{j}=\frac{l_{j+1}+\cdots+l_{n}}{T}, \quad j=\overline{1, n-1} .
$$

The polynomials $P \in \mathcal{P}_{n}^{\prime}(H)$ that have the same vector $\bar{s}=\left(l_{2}, \ldots, l_{n}\right)$ form a subclass which will be denoted by $\mathcal{P}_{n}(H, \bar{s})$.

Given $P \in \mathcal{P}_{n}^{\prime}(H)$, let

$$
S\left(\alpha_{i}\right)=\left\{x \in \mathbb{R}:\left|x-\alpha_{i}\right|=\min _{1 \leq j \leq n}\left|x-\alpha_{j}\right|\right\} .
$$

Lemma 1 (see [1]). If $P$ is a polynomial and $x \in S\left(\alpha_{1}\right)$, then

$$
\begin{gathered}
\left|x-\alpha_{1}\right| \leq 2^{n}|P(x)|\left|P^{\prime}\left(\alpha_{1}\right)\right|^{-1} \\
\left|x-\alpha_{1}\right| \leq \min _{2 \leq j \leq n}\left(2^{n-j}|P(x)|\left|P^{\prime}\left(\alpha_{1}\right)\right|^{-1} \prod_{k=2}^{j}\left|\alpha_{1}-\alpha_{k}\right|\right)^{1 / j} .
\end{gathered}
$$


LEMma 2. If $x \in S\left(\alpha_{1}\right)$, then

$$
\left|x-\alpha_{1}\right| \leq n \frac{|P(x)|}{\left|P^{\prime}(x)\right|} .
$$

Proof. Using the representation $P(x)=a_{n}\left(x-\alpha_{1}\right) \cdots\left(x-\alpha_{n}\right)$ we obtain

$$
\frac{\left|P^{\prime}(x)\right|}{|P(x)|} \leq \sum_{j=1}^{n} \frac{1}{\left|x-\alpha_{j}\right|} \leq \frac{n}{\left|x-\alpha_{1}\right|},
$$

whence the lemma readily follows.

Lemma 3 (see [7]). If $\left|a_{n}\right| \gg H(P)$, then all roots $\alpha_{j}$ of $P$ satisfy

$$
\left|\alpha_{j}\right| \ll 1 \text {. }
$$

In the next lemma we consider polynomials of fixed height $H$ only.

Lemma 4 (see [7]). Let $k, m \in \mathbb{Z}$ and $P \in \mathcal{P}_{n}^{\prime}(H)$. Then

$$
\max _{k \leq m \leq k+n}|P(m)|>c(n) H .
$$

Lemma 5 (see [2]). For any $n \in \mathbb{N}$ with $n>1$ and real $\delta>0$ there is an effectively computable bound $K_{0}(\delta, n)$ such that for any $K>K_{0}$ and positive real $\varsigma, \tau, \eta$ the following holds. If $P_{1}(x), P_{2}(x) \in \mathbb{Z}[x]$ are coprime and

$$
\max \left(\operatorname{deg} P_{1}, \operatorname{deg} P_{2}\right) \leq n, \quad \max \left(H\left(P_{1}\right), H\left(P_{2}\right)\right) \leq K^{\varsigma},
$$

and if there is an interval $I \subset \mathbb{R}$ with $|I|=K^{-\eta}$ such that

$$
\max \left(\left|P_{1}(x)\right|,\left|P_{2}(x)\right|\right)<K^{-\tau} \quad \text { for all } x \in I,
$$

then

$$
\tau+\varsigma+2 \max \{\tau+\varsigma-\eta, 0\}<2 n \varsigma+\delta .
$$

Lemma 6. Let $P \in \mathcal{P}_{n}^{\prime}(H)$ with $|D(P)|<Q^{2 n-2-2 v}$. Then there is a polynomial $T(x)=b_{n} x^{n}+b_{n-1} x^{n-1}+\cdots+b_{0}$ satisfying

$$
|D(T)|=|D(P)|, \quad H(T) \ll H \quad \text { and } \quad\left|b_{n}\right| \gg H .
$$

Proof. Let $\alpha_{1}, \ldots, \alpha_{n}$ be the roots of $P(x)=a_{n} x^{n}+\cdots+a_{1} x+a_{0}$. By Lemma 4 , there is an integer $m_{0}$ with $1 \leq m_{0} \leq n+1$ such that

$$
\left|P\left(m_{0}\right)\right|>c(n) H \text {. }
$$

Consider the polynomial $P_{1}(x)=P\left(x+m_{0}\right)=a_{n} x^{n}+a_{n-1}^{\prime} x^{n-1}+\cdots+$ $a_{1}^{\prime} x+P\left(m_{0}\right)$. Its roots are $\beta_{j}=\alpha_{j}-m_{0}, 1 \leq j \leq n$, and

$$
\left|D\left(P_{1}\right)\right|=a_{n}^{2 n-2}\left|\prod_{1 \leq i<j \leq n}\left(\beta_{i}-\beta_{j}\right)^{2}\right|=a_{n}^{2 n-2}\left|\prod_{1 \leq i<j \leq n}\left(\alpha_{i}-\alpha_{j}\right)^{2}\right|=|D(P)| .
$$

The polynomial $T(x)=x^{n} P_{1}(1 / x)=P\left(m_{0}\right) x^{n}+a_{n-1}^{\prime \prime} x^{n-1}+\cdots+a_{1}^{\prime \prime} x+a_{n}$ has roots $\gamma_{j}=1 / \beta_{j}=1 /\left(\alpha_{j}-m_{0}\right)$, and satisfies

$$
|D(T)|=P\left(m_{0}\right)^{2 n-2}\left|\prod_{1 \leq i<j \leq n}\left(\beta_{i}-\beta_{j}\right)^{2} \beta_{i}^{-2} \beta_{j}^{-2}\right| .
$$


But $\left|\prod_{1 \leq i<j \leq n} \beta_{i}^{-2} \beta_{j}^{-2}\right|=\left(P\left(m_{0}\right) a_{n}^{-1}\right)^{2 n-2}$, therefore $|D(T)|=|D(P)|$. The condition $H(T)<c(n) H$ is obviously satisfied because $H(T)=H\left(P_{1}\right)$ and $H\left(P_{1}\right) \asymp H(P)$.

Lemma 7 (see [1]). Let $P \in \mathcal{P}_{n}^{\prime}(H)$. Then

$$
\left|P^{(l)}\left(\alpha_{1}\right)\right| \ll H^{1-p_{l}}, \quad 1 \leq l \leq n-1 .
$$

Lemma 8 (see [2]). The measure of the set of those $x$ such that the inequality

$$
|P(x)|<H^{-w}
$$

for $w>n-1$ and $H>H_{0}$ has infinitely many solutions in reducible polynomials $P(x)$, tends to zero as $H_{0} \rightarrow \infty$.

4. Proof of Theorem 1 modulo Theorem 2. We shall show how Theorem 1 follows from Theorem 2 .

Suppose $P(x) \in \mathbb{Z}[x], \operatorname{deg} P \leq n,\left|a_{n}\right|>c H$.

Lemma 6 shows that the last inequality does not impose a restriction: if $\left|a_{n}\right| \leq c H$ then the polynomial can be transformed into a polynomial with a large highest coefficient without changing the value of its discriminant.

Using Dirichlet's principle we shall prove that for any $x \in I \subset \mathbb{R}$ and $Q>1$ there are two real positive numbers $c_{3}$ and $c_{4}$ with $\min \left(c_{3}, c_{4}\right) \leq 1$ and $c_{3} c_{4}>8 n$ such that the system of inequalities

$$
\left\{\begin{array}{l}
|P(x)|<c_{3} Q^{-n+v}, \\
\left|P^{\prime}(x)\right|<c_{4} Q^{1-v}, \quad H(P) \leq Q,
\end{array}\right.
$$

holds for some polynomials $P \in \mathcal{P}_{n}(Q)$.

Let $c_{3}=1, c_{4}=8 n$. Then system (7) may be rewritten as

$$
\left\{\begin{array}{l}
|P(x)|<Q^{-n+v} \\
\left|P^{\prime}(x)\right|<8 n Q^{1-v}
\end{array}\right.
$$

The existence of solutions to (8) and Theorem 2 implies that for $\gamma=$ $n^{-1} 2^{-n-12}$ the system of inequalities

$$
\left\{\begin{array}{l}
\gamma Q^{-n+v}<|P(x)|<Q^{-n+v} \\
\gamma Q^{1-v}<\left|P^{\prime}(x)\right|<8 n Q^{1-v}
\end{array}\right.
$$

has solutions in $P \in \mathcal{P}_{n}(Q)$ for all $x \in B_{1}$ with $\mu B_{1} \geq|I| / 2$. Indeed, if one of the inequalities in (9) does not hold then $|P(x)| \leq \gamma Q^{-n+v},\left|P^{\prime}(x)\right|<$ $8 n Q^{-1-v}$ and $8 n \gamma<2^{-n-9}$. If $\left|P^{\prime}(x)\right|<\gamma Q^{1-v},|P(x)| \leq Q^{-n+v}$ then $c_{1} c_{2}<$ $n^{-1} 2^{-n-12}$. The claim reduces to the fact that the system of inequalities does not hold on the set $B$ with measure $\mu B<|I| / 2$ and holds for all $x \in B_{1}=I \backslash B$ with $\mu B_{1} \geq|I| / 2$.

Let us choose $x_{1} \in B_{1}$. Then we can find a polynomial $P_{1}(x)$ for which system (9) holds for $x=x_{1}$. For all $x$ in the interval $\left|x-x_{1}\right|<Q^{-2 / 3}$, the 
Mean Value Theorem gives

$$
P_{1}^{\prime}(x)=P_{1}^{\prime}\left(x_{1}\right)+P_{1}^{\prime \prime}\left(\xi_{1}\right)\left(x-x_{1}\right) \quad \text { for some } \xi_{1} \in\left[x, x_{1}\right] .
$$

The obvious estimate $\left|P^{\prime \prime}\left(\xi_{2}\right)\right|<n^{3} Q$ implies $\left|P^{\prime \prime}\left(\xi_{1}\right)\left(x-x_{1}\right)\right|<n^{3} Q^{1 / 3}$. But $\left|P_{1}^{\prime}\left(x_{1}\right)\right| \gg Q^{1 / 2}$ for $v \leq 1 / 2$ and therefore for sufficiently large $Q$ it follows from (10) and the second inequality in (9) that

$$
\frac{1}{2} \gamma Q^{1-v}<\frac{1}{2}\left|P_{1}^{\prime}\left(x_{1}\right)\right|<\left|P_{1}^{\prime}(x)\right|<2\left|P_{1}^{\prime}\left(x_{1}\right)\right|<16 n Q^{1-v} .
$$

In view of the values of $P\left(x_{1}\right)$ and $P^{\prime}\left(x_{1}\right)$ given by (9) we can distinguish four possible combinations of signs. We will consider the case when $P_{1}\left(x_{1}\right)$ $<0$ and $P_{1}^{\prime}\left(x_{1}\right)>0$. The remaining ones can be dealt with in a similar way. Again we use the Mean Value Theorem:

$$
P_{1}(x)=P_{1}\left(x_{1}\right)+P_{1}^{\prime}\left(\xi_{2}\right)\left(x-x_{1}\right) \quad \text { for some } \xi_{2} \in\left[x_{1}, x\right] .
$$

Write $x=x_{1}+\Delta$ and suppose that $\Delta>2 \gamma^{-1} Q^{-n-1+2 v}$. If $P_{1}\left(x_{1}\right)<$ $P_{1}\left(x_{1}+\Delta\right)<0$ then the first inequality of (9) implies

$$
0<P_{1}\left(x_{1}+\Delta\right)-P_{1}\left(x_{1}\right)<Q^{-n+v} .
$$

On the other hand, we have

$$
\left|P^{\prime}\left(\xi_{2}\right) \Delta\right|>\frac{1}{2} \gamma Q^{1-v} 2 \gamma^{-1} Q^{-n-1+2 v}=Q^{-n+v} .
$$

We thus obtain a contradiction to (11). This means that $P_{1}\left(x_{1}+\Delta\right)>0$ and there is a real root $\alpha$ of $P_{1}(x)$ between $x_{1}$ and $x_{1}+\Delta$.

At the same time

$$
\left|x_{1}-\alpha\right|<2 \gamma^{-1} Q^{-n-1+2 v}=n 2^{n+13} Q^{-n-1+2 v} .
$$

Now we shall obtain a lower bound for $\left|x_{1}-\alpha\right|$. Again we consider only one of four possibilities, $P_{1}\left(x_{1}\right)>0, P_{1}^{\prime}\left(x_{1}\right)<0$. At $x=x_{1}+\Delta_{1}$ we have

$$
P_{1}(x)=P_{1}\left(x_{1}\right)+P_{1}^{\prime}\left(\xi_{3}\right) \Delta_{1} \quad \text { for some } \xi_{3} \in\left[x_{1}, x\right] .
$$

If $\Delta_{1}<2^{-4} n^{-1} \gamma Q^{-n-1+2 v}$ then $\left|P_{1}\left(x_{1}\right)\right|>\gamma Q^{-n+v}$ and $\left|P^{\prime}\left(\xi_{3}\right) \Delta_{1}\right|<$ $\gamma Q^{-n+v}$. Then (13) implies that $P_{1}(x)$ cannot have any root in $\left[x_{1}, x_{1}+\Delta_{1}\right]$ and therefore for any root $\alpha$, we have

$$
n^{-1} 2^{-n-13} Q^{-n-1+2 v}<|x-\alpha| .
$$

Let $\alpha$ be the root of $P_{1}(x)$ closest to $x_{1}$. Using the representation

$$
P_{1}^{\prime}(\alpha)=P_{1}^{\prime}\left(x_{1}\right)+P_{1}^{\prime \prime}\left(\xi_{4}\right)\left(x_{1}-\alpha\right) \quad \text { for some } \xi_{4} \in[x, \alpha],
$$

the estimate $\left|P_{1}^{\prime \prime}(\xi)\right|<n^{3} Q$ and (12) for sufficiently large $Q$ we get

$$
n^{-1} 2^{-n-13} Q^{1-v}<\left|P_{1}^{\prime}(\alpha)\right|<16 n Q^{1-v} .
$$

The square of derivative is a factor of the discriminant of $P$. Taking into account that for $\left|a_{n}\right| \asymp H(P)$ all roots of the polynomial are bounded (see 
Lemma 3) we can estimate the differences $\left|\alpha_{i}-\alpha_{j}\right|, 2 \leq i<j \leq n$, by a constant $c(n)$. Thus, for $x_{1} \in B_{1}$ we can construct a polynomial $P_{1}(x)$ with

$$
\left|D\left(P_{1}\right)\right| \ll Q^{2 n-2-2 v} .
$$

Define $x_{01}=\inf \left\{x: x \in I \cap B_{1}\right\}$. Clearly $x_{1} \in B_{1}$ can be taken from the interval $J_{1}=\left[x_{01}, x_{01}+Q^{-n-1}\right]$. Set $J_{1}^{\prime}=\left[x_{01}, x_{01}+Q^{-n-1}+4 \gamma^{-1} Q^{-n-1+2 v}\right]$ and $x_{02}=\inf \left\{x: x \in\left(I \backslash J_{1}^{\prime}\right) \cap B_{1}\right\}$. Choose $x_{2} \in J_{2}=\left[x_{02}, x_{02}+Q^{-n-1}\right] \cap B_{1}$. By construction, we have

$$
\left|x_{2}-x_{1}\right|>4 \gamma^{-1} Q^{-n-1+2 v} .
$$

For this point we can construct a polynomial $P_{2}(x)$ again satisfying the system of inequalities $(9)$ at $x_{2}$. We will show that $P_{2}(x) \neq P_{1}(x)$. Consider the value of the polynomial $P_{1}(x)$ at $x=x_{2}$. Then

$$
P_{1}\left(x_{2}\right)=P_{1}\left(x_{1}\right)+P_{1}^{\prime}\left(\xi_{5}\right)\left(x_{2}-x_{1}\right) \quad \text { for some } \xi_{5} \in\left[x_{1}, x_{2}\right] .
$$

Using $\left|P_{1}\left(x_{1}\right)\right|<Q^{-n+v},\left|P_{1}^{\prime}\left(\xi_{5}\right)\right|>(\gamma / 2) Q^{1-v}$ and (14) we obtain

$$
\left|P_{1}\left(x_{2}\right)\right|>Q^{-n+v}
$$

so $P_{1}$ does not satisfy the first inequality of (9). Thus, $P_{2}(x)$ is different from $P_{1}(x)$ at $x_{2}$. The discriminant $D\left(P_{2}\right)$ also satisfies (5). Moreover, for a point $x_{3} \in B_{1}$ with $x_{3}-x_{2}>4 \gamma^{-1} Q^{-n-1+2 v}$, we construct a polynomial $P_{3}(x)$ different from $P_{1}(x)$ and $P_{2}(x)$ that satisfies conditions (5) and (9). It is clear that repeating the described procedure we can construct $c(n) Q^{n+1-2 v}$ polynomials $P(x)$ with discriminants satisfying (5).

5. Proof of Theorem 2. We start by estimating the measure of the set of those $x$ such that the system

$$
\left\{\begin{array}{l}
|P(x)|<c_{1} Q^{-n+v} \\
Q^{1-v_{1}}<\left|P^{\prime}(x)\right|<c_{2} Q^{1-v}
\end{array}\right.
$$

is solvable in $P$, where $v_{1}$ with $v<v_{1}<1$ will be specified later.

We shall show that $P^{\prime}(x)$ in the second inequality of (15) can be replaced by $P^{\prime}(\alpha)$, where $\alpha$ denotes the root of $P$ nearest to $x$. Using the Mean Value Theorem gives

$$
P^{\prime}(x)=P^{\prime}(\alpha)+P^{\prime \prime}\left(\xi_{1}\right)(x-\alpha) \quad \text { for some } \xi_{1} \in(\alpha, x) .
$$

By Lemma 2,

$$
|x-\alpha|<n \frac{|P(x)|}{\left|P^{\prime}(x)\right|}
$$

Then

$$
\left|P^{\prime}(\alpha)\right|=\left|P^{\prime}(x)-P^{\prime \prime}\left(\xi_{1}\right)(x-\alpha)\right|
$$

As

$$
\left|P^{\prime \prime}\left(\xi_{1}\right)(x-\alpha)\right| \leq n^{3} Q c_{1} n Q^{-n-1+v+v_{1}}=c_{1} n^{4} Q^{-n+v+v_{1}}
$$


for sufficiently large $Q$ we obtain

$$
\frac{3}{4} Q^{1-v_{1}} \leq \frac{3}{4}\left|P^{\prime}(x)\right| \leq\left|P^{\prime}(\alpha)\right| \leq \frac{4}{3}\left|P^{\prime}(x)\right| \leq \frac{4}{3} c_{2} Q^{1-v}
$$

and

$$
\frac{3}{4}\left|P^{\prime}(\alpha)\right| \leq\left|P^{\prime}(x)\right| \leq \frac{4}{3}\left|P^{\prime}(\alpha)\right| .
$$

Therefore for sufficiently large $Q,(15)$ implies

$$
\left\{\begin{array}{l}
|P(x)|<c_{1} Q^{-n+v}, \\
\frac{3}{4} Q^{1-v_{1}}<\left|P^{\prime}(\alpha)\right|<\frac{4}{3} c_{2} Q^{1-v}, \\
\left|a_{j}\right| \leq Q .
\end{array}\right.
$$

Let $\mathcal{L}_{n}^{\prime}(v)$ denote the set of $x$ for which the system (16) is solvable in $P$. Now we will prove that $\mu \mathcal{L}_{n}^{\prime}(v)<\frac{3}{8}|I|$.

Consider the intervals

$$
\sigma_{1}(P): \quad|x-\alpha|<\frac{4}{3} c_{1} n Q^{-n+v}\left|P^{\prime}(\alpha)\right|^{-1}
$$

and

$$
\sigma_{2}(P): \quad|x-\alpha|<c_{5} Q^{-1+v}\left|P^{\prime}(\alpha)\right|^{-1} .
$$

The value of $c_{5}$ will be specified below. Obviously

$$
\left|\sigma_{1}(P)\right| \leq \frac{4}{3} c_{1} c_{5}^{-1} n Q^{-n+1}\left|\sigma_{2}(P)\right| .
$$

Fix the vector $\bar{b}=\left(a_{n}, \ldots, a_{2}\right)$ of coefficients of $P(x)$. The polynomials $P \in \mathcal{P}_{n}(Q)$ with the same vector $\bar{b}$ form a subclass of $\mathcal{P}_{n}(Q)$ which will be denoted by $\mathcal{P}(\bar{b})$.

The interval $\sigma_{2}\left(P_{1}\right)$ with $P_{1} \in \mathcal{P}(\bar{b})$ is called inessential if there is another interval $\sigma_{2}\left(P_{2}\right)$ with $P_{2} \in \mathcal{P}(\bar{b})$ such that

$$
\left|\sigma_{2}\left(P_{1}\right) \cap \sigma_{2}\left(P_{2}\right)\right| \geq 0.5\left|\sigma_{2}\left(P_{1}\right)\right| .
$$

Otherwise for any $P_{2} \in \mathcal{P}(\bar{b})$ different from $P_{1}$,

$$
\left|\sigma_{2}\left(P_{1}\right) \cap \sigma_{2}\left(P_{2}\right)\right|<0.5\left|\sigma_{2}\left(P_{1}\right)\right|
$$

and the interval $\sigma_{2}\left(P_{2}\right)$ is called essential.

The case of essential intervals. In this case every point $x \in I$ belongs to no more than two essential intervals $\sigma_{2}(P)$. Hence for any vector $\bar{b}$,

$$
\sum_{P \in \mathcal{P}_{1}(\bar{b})}\left|\sigma_{2}(P)\right| \leq 2|I| .
$$

We have to sum over the lengths of the essential intervals $\sigma_{1}(P)$ inside the class $\mathcal{P}(\bar{b})$ with fixed vector $\bar{b}$, and then over all classes. We can estimate the number of classes as the number of all possible vectors $\bar{b}$,

$$
(2 Q+1)^{n-1}=(2 Q)^{n-1}\left(1+\frac{1}{2 Q}\right)^{n-1} \leq 2^{n-1} Q^{n-1} e^{(n-1) / 2 Q}<2^{n} Q^{n-1} .
$$


From (17) and (18) we obtain

$$
\sum_{\bar{b},\left|a_{j}\right| \leq Q} \sum_{P \in \mathcal{P}(\bar{b})}\left|\sigma_{1}(P)\right|<\frac{4}{3} c_{1} c_{5}^{-1} n Q^{-n+1} 2|I| 2^{n} Q^{n-1}=n 2^{n+2} c_{1} c_{5}^{-1} .
$$

Thus for $c_{5}=n 2^{n+5} c_{1}$ the measure will be no larger than $|I| / 8$.

The case of inessential intervals. Let us estimate the values of $\left|P_{j}(x)\right|$, $j=1,2$, on the intersection $\sigma_{2}\left(P_{1}, P_{2}\right)$ of the intervals $\sigma_{2}\left(P_{1}\right)$ and $\sigma_{2}\left(P_{2}\right)$. By the Mean Value Theorem,

$$
P_{j}(x)=P_{j}^{\prime}(\alpha)(x-\alpha)+\frac{1}{2} P_{j}^{\prime \prime}\left(\xi_{2}\right)(x-\alpha)^{2} \quad \text { for some } \xi_{2} \in(\alpha, x),
$$

and

$$
P_{j}^{\prime}(x)=P_{j}^{\prime}(\alpha)+P_{j}^{\prime \prime}\left(\xi_{3}\right)(x-\alpha) \quad \text { for some } \xi_{3} \in(\alpha, x) .
$$

The second summand is estimated by

while

$$
\left|P^{\prime \prime}\left(\xi_{2}\right)(x-\alpha)^{2}\right| \leq 2 n^{3} c_{5}^{2} Q^{-3+2 v+2 v_{1}},
$$

$$
\left|P^{\prime}(\alpha)(x-\alpha)\right|<c_{5} Q^{-1+v} .
$$

As $2 v_{1}<2-v$ for an appropriate choice of $v_{1}<3 / 4$ we obtain

$$
\left|P_{j}(x)\right| \leq \frac{4}{3} c_{5} Q^{-1+v}, \quad j=1,2 .
$$

Similarly we obtain the following estimates:

$$
\left|P_{j}^{\prime}(\alpha)\right|<\frac{4}{3} c_{2} Q^{1-v}, \quad\left|P_{j}^{\prime \prime}\left(\xi_{3}\right)(x-\alpha)\right|<2 n^{3} c_{5} Q^{-1+v+v_{1}}
$$

and for $v_{1} \leq 1$,

$$
\left|P_{j}^{\prime}(x)\right| \leq \frac{4}{3} c_{2} Q^{1-v}, \quad j=1,2 .
$$

Define $K(x)=P_{2}(x)-P_{1}(x)$. Obviously $K(x)$ is not identically zero and has the form $K(x)=b_{1} x+b_{0}$. Moreover, (19) and (20) imply

$$
\left|b_{1} x+b_{0}\right|<\frac{8}{3} c_{5} Q^{-1+v}
$$

and

$$
\left|b_{1}\right|=\left|K^{\prime}(x)\right|<\frac{8}{3} c_{2} Q^{1-v} .
$$

For fixed $b_{0}$ and $b_{1}$ the measure of those $x \in I$ that satisfy (21) does not exceed $\frac{16}{3} c_{5} Q^{-\lambda} b_{1}^{-1}$. Given that $x \in I$ and (21) is satisfied we find that $b_{0}$ can have not more than $|I|\left|b_{1}\right|+2$ values. Summing over all $b_{0}$ we obtain an estimate for the measure when $b_{1}$ is fixed,

$$
\frac{16}{3} c_{5} Q^{-1+v} b_{1}^{-1}(|I||b|+2)<6 c_{5} Q^{-1+v} .
$$

After summing (22) over all $\left|b_{1}\right|$ we have

$$
2^{5} c_{2} c_{5} Q^{1-v-\lambda}|I|=n 2^{n+8} c_{1} c_{2}|I|=\frac{1}{8}|I| .
$$

From $c_{1} c_{2}<n^{-1} 2^{-n-11}$ we can estimate the total measure for both essential and inessential intervals by $|I| / 4$.

Now we consider the remaining case. Our task is to estimate the measure of $\mathcal{L}_{n}^{\prime \prime}(v)$, the set of all $x$ such that the system 


$$
\left\{\begin{array}{l}
|P(x)|<Q^{-n+v}, \\
\left|P^{\prime}(x)\right|<Q^{1-v_{1}}, \\
\left|a_{j}\right| \leq Q
\end{array}\right.
$$

is solvable in $P \in \mathcal{P}_{n}(Q)$.

To prove Theorem 2 it remains to show that

$$
\mu \mathcal{L}_{n}^{\prime \prime}(v) \ll \frac{1}{4}|I| .
$$

The proof splits into the following cases:

1. $l_{2} T^{-1}+p_{1} \geq n+1-v$,

2. $n+0.1 \leq l_{2} T^{-1}+p_{1}<n+1-v$,

3. $7 / 4 \leq l_{2} T^{-1}+p_{1}<n+0.1$,

4. $l_{2} T^{-1}+p_{1}<7 / 4$.

CASE 1:

$$
l_{2} T^{-1}+p_{1} \geq n+1-v .
$$

Consider the class $\mathcal{P}_{t}(\bar{s})=\bigcup_{2^{t}<H<2^{t+1}} \mathcal{P}_{n}(H, \bar{s})$. Since $Q$ is a sufficiently large number and $H \leq Q$, we have $t_{0}<t \ll \log Q$. We are going to compare two estimates for $\left|x-\alpha_{1}\right|$ obtained from (23) and Lemma 1 for $x \in S\left(\alpha_{1}\right)$,

$$
\left|x-\alpha_{1}\right| \leq 2^{n} \frac{|P(x)|}{\left|P^{\prime}\left(\alpha_{1}\right)\right|} \ll 2^{t\left(-n+v-1+p_{1}+(n-1) \varepsilon\right)}
$$

and

$$
\left|x-\alpha_{1}\right| \leq\left(2^{n-1} \frac{|P(x)|\left|\alpha_{1}-\alpha_{2}\right|}{\left|P^{\prime}\left(\alpha_{1}\right)\right|}\right)^{1 / 2} \ll 2^{t\left(-n+v-1+p_{2}+(n-2) \varepsilon\right) / 2} .
$$

In the case (24) we use the estimate (26). Let us divide the interval $I$ into smaller parts $I_{j}$ with $\mu I_{j}=2^{-t\left(\left(n+1-v-p_{2}\right) / 2-\gamma\right)}$, where $\gamma$ is a positive constant.

For an integral polynomial $P(x)$ and an interval $I_{j}$ we shall write " $P(x)$ belongs to $I_{j}$ " or " $I_{j}$ contains $P(x)$ " if there is a point $x \in I_{j}$ that satisfies the system (23). Let $\sigma(P)$ denote the measure of $x \in S\left(\alpha_{1}\right)$ satisfying (23).

(a) Assume that there is at most one polynomial $P \in \mathcal{P}_{t}(\bar{s})$ that belongs to every $I_{j}$. Then for every polynomial the measure of the set of those $x$ that satisfy (23) does not exceed $c(n) 2^{-t\left(n+1-v-p_{2}-(n-2) \varepsilon\right) / 2}$ and the number of $I_{j}$ is less than $2^{t\left(\left(n+1-v-p_{2}\right) / 2-\gamma\right)}|I|$. Therefore

$$
\begin{aligned}
& \sum_{P \in \mathcal{P}_{t}(\bar{s})} \sigma(P) \\
& \ll \sum_{P \in \mathcal{P}_{t}(\bar{s})} 2^{t\left(\left(n+1-v-p_{2}\right) / 2-\gamma\right)}|I| \cdot c(n) 2^{-t\left(n+1-v-p_{2}-(n-2) \varepsilon\right) / 2} \ll 2^{-t \gamma_{1}},
\end{aligned}
$$

where $\gamma_{1}=\gamma-(n-2) \varepsilon / 2$. 
The sum (27) extends over all $t \geq t_{0}$. Since $\sum_{t>t_{0}} 2^{-t \gamma_{1}} \ll 2^{-t_{0} \gamma_{1}}$, for sufficiently large $t_{0}$ the measure of the set of those $x$ such that the system (23) holds and polynomials $P(x)$ satisfy Case 1 (a) does not exceed $|I| / 32$.

(b) Suppose the contrary, that there are intervals $I_{j}$ that contain at least two polynomials, i.e. we can find polynomials $P_{1}$ and $P_{2}$ from the class $\mathcal{P}_{t}(\bar{s})$, and points $x_{1}$ and $x_{2}$ from $I_{j}$, that satisfy the system of inequalities

$$
\left\{\begin{array} { l } 
{ | P _ { 1 } ( x _ { 1 } ) | \ll 2 ^ { t ( - n + v ) } , } \\
{ | P _ { 1 } ^ { \prime } ( x _ { 1 } ) | \ll 2 ^ { t ( 1 - v _ { 1 } ) } , }
\end{array} \quad \left\{\begin{array}{l}
\left|P_{2}\left(x_{2}\right)\right| \ll 2^{t(-n+v)}, \\
\left|P_{2}^{\prime}\left(x_{2}\right)\right| \ll 2^{t\left(1-v_{1}\right)} .
\end{array}\right.\right.
$$

Let us estimate the value of $P_{1}(x)$ and $P_{2}(x)$ at points of the interval $I_{j}$. Using Taylor's expansion for $P_{i}(x)$ at $\alpha_{1}$,

$$
P_{i}(x)=\sum_{j=1}^{n} \frac{P_{i}^{(j)}\left(\alpha_{1}\right)\left(x-\alpha_{1}\right)^{j}}{j !},
$$

and estimates $\left|P^{j}\left(\alpha_{1}\right)\right|$ from Lemmas 1 and 7 we get

$$
\left|P_{j}(x)\right| \ll 2^{t\left(1-p_{j}+j\left(\left(-n+v-1+p_{j}+(n-j) \varepsilon\right) / j+\gamma\right)\right)} \ll 2^{t\left(-n+v+n \gamma_{1}\right)} .
$$

Now for polynomials $P_{1}$ and $P_{2}$ without common roots we can apply Lemma 5.

Since we have $\tau=n-v-n \gamma_{1}, \varsigma=1, \eta=\left(n+1-v-p_{2}\right) / 2-\gamma$, it follows that

$$
n-v-n \gamma_{1}+1+2\left(n-v-n \gamma_{1}+1-\frac{n+1-v-p_{2}}{2}+\gamma\right)<2 n+\delta .
$$

Hence

$$
2-2 v<\delta+(3 n-2) \gamma_{1} .
$$

The latter leads to a contradiction for $v \leq 1 / 2$ and sufficiently small $\gamma, \varepsilon$ and $\delta$.

CASE 2:

$$
n+0.1 \leq l_{2} T^{-1}+p_{1}<n+1-v .
$$

Let us divide the interval $I$ into intervals $I_{j}$, where $\left|I_{j}\right|=2^{t\left(-l_{2} / T+\gamma\right)}$.

(a) Assume that no more than one polynomial $P \in \mathcal{P}_{t}(\bar{s})$ belongs to every $I_{j}$. We use inequality (25). For every polynomial the measure of the set of $x$ 's satisfying $(23)$ does not exceed $c(n) 2^{-t\left(n+1-v-p_{1}-(n-1) \varepsilon\right)}$. Further, the number of $I_{j}$ is less than $2^{t\left(n+1-v-p_{1}-\gamma\right)}|I|$. Therefore

$$
\sum_{P \in \mathcal{P}_{t}(\bar{s})} \sigma(P) \ll \sum_{P \in \mathcal{P}_{t}(\bar{s})} 2^{t\left(l_{2} / T-\gamma\right)} \cdot 2^{-t\left(n+1-v-p_{1}-(n-1) \varepsilon\right)} \ll 2^{-t \gamma_{2}},
$$

where $\gamma_{2}=\gamma-(n-1) \varepsilon$. Again we sum the estimate (29) over all $t>t_{0}$ as in formula (27). It is clear that the total sum is less than $|I| / 32$. 
(b) Assuming, as in Case 1 above, the existence of an interval $I_{j}$ that contains at least two different polynomials $P_{1}(x)$ and $P_{2}(x)$, for any $x \in I_{j}$ by Taylor's expansion we get

$$
\left|P_{i}(x)\right| \ll 2^{-t\left(l_{2} T^{-1}+p_{1}-1-2 \gamma\right)}, \quad i=1,2 .
$$

For $P_{1}(x)$ and $P_{2}(x)$ which have no common roots, on $I_{j}$ we may apply Lemma 5 with $\varsigma=1, \eta=l_{2} T^{-1}-\gamma, \tau+1=l_{2} T^{-1}+p_{1}-2 \gamma$. Note that $l_{2} T^{-1} \leq p_{1}$. Then

$$
l_{2} T^{-1}+3 p_{1}-4 \gamma<2 n+\delta .
$$

This together with (28) implies the inequalities

$$
2 n+\frac{1}{5}-4 \gamma \leq l_{2} T^{-1}+3 p_{1}-4 \gamma<2 n-\delta,
$$

so

$$
\frac{1}{5}<\delta+4 \gamma
$$

which are contradictory for small $\delta$ and $\gamma$.

CASE 3:

$$
\frac{7}{4} \leq l_{2} T^{-1}+p_{1}<n+\frac{1}{10} .
$$

This case represents the largest interval for $l_{2} T^{-1}+p_{1}$ and is the most difficult. We divide $I$ into intervals $I_{j}$ of length $2^{-t l_{2} T^{-1}}$. First let us estimate the value of a polynomial $P \in \mathcal{P}_{n}$ and its derivative on $I_{j}$. For this purpose expand by Taylor's formula, in the neighborhood of $\alpha_{1}$,

$$
\begin{gathered}
P(x)=\sum_{j=1}^{n} \frac{P^{(j)}\left(\alpha_{1}\right)\left(x-\alpha_{1}\right)^{j}}{j !}, \\
\left|P^{\prime}\left(\alpha_{1}\right)\left(x-\alpha_{1}\right)\right| \ll 2^{t\left(1-p_{1}-l_{2} T^{-1}\right)}, \\
\left|P^{\prime \prime}\left(\alpha_{1}\right)\left(x-\alpha_{1}\right)^{2}\right| \ll 2^{t\left(1-p_{2}-2 l_{2} T^{-1}\right)} \ll 2^{t\left(1-p_{1}-l_{2} T^{-1}\right)}, \\
\left|P^{(i)}\left(\alpha_{1}\right)\left(x-\alpha_{1}\right)^{i}\right| \ll 2^{t\left(1-p_{i}-i l_{2} T^{-1}\right)} \ll 2^{t\left(1-p_{1}-l_{2} T^{-1}\right)}, \quad 3 \leq i \leq n .
\end{gathered}
$$

Similarly we treat the derivative:

$$
\begin{gathered}
P^{\prime}(x)=\sum_{j=0}^{n-1} \frac{P^{(j+1)}\left(\alpha_{1}\right)\left(x-\alpha_{1}\right)^{j}}{j !}, \\
\left|P^{\prime}\left(\alpha_{1}\right)\right| \asymp 2^{t\left(1-p_{1}\right)}, \\
\left|P^{(i)}\left(\alpha_{1}\right)\left(x-\alpha_{1}\right)^{i-1}\right| \ll 2^{t\left(1-p_{i}-(i-1) l_{2} T^{-1}\right)} \ll 2^{t\left(1-p_{1}\right)}, \quad 2 \leq i \leq n .
\end{gathered}
$$

Thus, if the polynomial $P(x)$ belongs to the interval $I_{j}$ it should satisfy the system

$$
\left\{\begin{array}{l}
|P(x)| \ll 2^{t\left(1-p_{1}-l_{2} t^{-1}\right)}, \\
\left|P^{\prime}(x)\right| \asymp 2^{t\left(1-p_{1}\right)} .
\end{array}\right.
$$


Consider those intervals that contain $c(n) 2^{\text {te }}$ polynomials. Then the measure of the set of $x \in I$ that satisfy (23) is

$$
2^{t\left(-n+v-1+p_{1}+(n-1) \varepsilon\right)} c(n) 2^{t \varrho} 2^{t l_{2} T^{-1}} .
$$

If $\varrho<n+1-v-\left(p_{1}+l_{2} T^{-1}\right)$ and $t>t_{0}$ the measure can be estimated by $|I| / 32$.

To simplify subsequent computations we introduce

$$
u:=n+1-v-p_{1}-l_{2} T^{-1} .
$$

It follows from (30) and $v \leq 1 / 2$ that $u \geq 2 / 5$. Let $u_{1}=u-1 / 5 \geq 1 / 5$ and represent $u_{1}$ as the sum $u_{1}=\left[u_{1}\right]+\left\{u_{1}\right\}$.

Let $n+1-v-p_{1}-\varrho-l_{2} T^{-1} \leq 0$, i.e. $\varrho \geq u$. By Dirichlet's principle, there exist at least $c(n) 2^{t\left(\left\{u_{1}\right\}+0.2\right)}$ polynomials $P_{1}(x), \ldots, P_{k}(x)$, where $k \gg$ $2^{t\left(\left\{u_{1}\right\}+0.2\right)}$ such that the first $\left[u_{1}\right]$ coefficients are identical.

Consider the polynomials $R_{j}(x)=P_{j+1}(x)-P_{1}(x)$, which obviously satisfy

$$
\operatorname{deg} R_{j}(x) \leq n-\left[u_{1}\right], \quad H\left(R_{j}\right) \ll 2^{t} .
$$

From (31) we get

$$
\left\{\begin{array}{l}
\left|R_{j}(x)\right| \ll 2^{t\left(1-p_{1}-l_{2} T^{-1}\right)}, \quad j=1, \ldots, k, \\
\left|R_{j}^{\prime}(x)\right| \ll 2^{t\left(1-p_{1}\right)} .
\end{array}\right.
$$

Every coefficient of the polynomial $R_{j}$ ranges in the interval $\left[-2^{t+1}, 2^{t+1}\right]$. We divide all intervals into equal parts of length $2^{t \vartheta}$, where $\vartheta=1-$ $\left\{u_{1}\right\} /\left(n-\left[u_{1}\right]\right)$.

Then at least $c(n) 2^{t / 5}$ polynomials fall into the same intervals. Hence the height of their differences $R_{j}(x)$ will be less than

$$
c(n) 2^{t \vartheta}=c(n) 2^{t\left(1-\left\{u_{1}\right\} /\left(n-\left[u_{1}\right]\right)\right)} .
$$

Define $S_{j}(x)=R_{j+1}(x)-R_{1}(x)$ and rewrite (32) as follows:

$$
\left\{\begin{array}{l}
\left|S_{j}(x)\right| \ll 2^{t\left(1-p_{1}-l_{2} \cdot T^{-1}\right)}, \quad j=1, \ldots, k-1, \\
\left|S_{j}^{\prime}(x)\right| \ll 2^{t\left(1-p_{1}\right)}, \quad j=1, \ldots, k-1, \\
\operatorname{deg} S_{j} \leq n-\left[u_{1}\right], \\
H\left(S_{i}\right)<2^{t\left(1-\left\{u_{1}\right\} /\left(n-\left[u_{1}\right]-1\right)\right)}
\end{array}\right.
$$

(a) Suppose there are coprime polynomials of type $S_{i}(x)$. Then applying Lemma 5 with $I=I_{j}$ and

$$
\begin{gathered}
\tau=p_{1}+l_{2} T^{-1}-1, \quad \vartheta=1-\frac{\left\{u_{1}\right\}}{n-\left[u_{1}\right]}, \\
\max \left\{\operatorname{deg} S_{1}, \operatorname{deg} S_{2}\right\} \leq \operatorname{deg} S=n-\left[u_{1}\right], \quad \eta=l_{2} T^{-1},
\end{gathered}
$$


we get

$$
\begin{aligned}
p_{1}+l_{2} T^{-1}-\frac{\left\{u_{1}\right\}}{n-\left[u_{1}\right]}+2\left(p_{1}+l_{2} T^{-1}\right. & \left.-\frac{\left\{u_{1}\right\}}{n-\left[u_{1}\right]}-l_{2} T^{-1}\right) \\
& \leq 2\left(n-\left[u_{1}\right]\right)\left(1-\frac{\left\{u_{1}\right\}}{n-\left[u_{1}\right]}\right)+\delta .
\end{aligned}
$$

This implies

$$
3 p_{1}-l_{2} T^{-1}-\frac{3\left\{u_{1}\right\}}{n-\left[u_{1}\right]} \leq 2 p_{1}+2 l_{2} T^{-1}+2 v+0.4-2+\delta .
$$

Replacing $p_{1}$ by $l_{2} T^{-1}$ and representing $n-\left[u_{1}\right]-1$ as $p_{1}+l_{2} T^{-1}+0.2+$ $\left\{u_{1}\right\}-1$ we obtain

$$
\frac{8}{5}-2 v<\frac{3\left\{u_{1}\right\}}{p_{1}+l_{2} T^{-1}+v+0.2+\left\{u_{1}\right\}-1}+\delta .
$$

By writing the right hand side of the inequality as a function of $\left\{u_{1}\right\}$ and $v$ in $[0 ; 1) \times[0 ; 1 / 4)$, we show that it does not exceed $0.4+3 /\left(p_{1}+l_{2} T^{-1}+0.2\right)$ $+\delta$, but our assumption $p_{1}+l_{2} T^{-1}>1$ leads to a contradiction to (34) for small enough $\delta$.

(b) If all the polynomials $S_{j}(x)$ are of the type $l S_{0}(x)$ then $\left|2^{0.4 t} S_{0}(x)\right| \ll$ $2^{t\left(1-p_{1}-l_{2} T^{-1}\right)}$ and

$$
\left|S_{0}(x)\right| \ll H\left(S_{0}\right)^{\frac{1-p_{1}-l_{2} T^{-1}-0.2}{1-\left\{u_{1}\right\} /\left(n-\left[u_{1}\right]\right)-0.2}} .
$$

If the inequality

$$
p_{1}+l_{2} T^{-1}+0.2-1>\left(n-\left[u_{1}\right]\right)\left(1-\frac{\left\{u_{1}\right\}}{n-\left[u_{1}\right]}-0.2\right)
$$

is false then Sprindžuk's theorem [7] implies that in Case 2(b) we can estimate the measure by $|I| / 32$.

Since the product on the right side of $(35)$ is $p_{1}+l_{2} T^{-1}-0.8$, the inequality (35) leads to a contradiction.

(c) If there is a reducible polynomial $S_{i}(x)$ decomposing into the product $S_{i}(x)=T_{1}(x) T_{2}(x)$ then system (33) implies that

$$
\left\{\begin{array}{l}
\left|T_{1}(x)\right| \ll H\left(T_{1}\right)^{\left(1-p_{1}-l_{2} T^{-1}\right)\left(1-\left\{u_{1}\right\} /\left(n-\left[u_{1}\right]\right)\right)^{-1}}, \\
\operatorname{deg} T \leq n-\left[u_{1}\right]-1 .
\end{array}\right.
$$

To apply Sprindžuk's theorem we verify the inequality

$$
p_{1}+l_{2} T^{-1}-1>\left(n-\left[u_{1}\right]-1\right)\left(1-\frac{\left\{u_{1}\right\}}{n-\left[u_{1}\right]}\right) .
$$

To this end rewrite the right hand side of (36) as

$$
p_{1}+l_{2} T^{-1}+0.2-2+\frac{1}{p_{1}+l_{2} T^{-1}+0.2} \text {. }
$$


Determining the maximum of this expression as the function of $\left\{u_{1}\right\}$ we find that the right side does not exceed

$$
p_{1}+l_{2} T^{-1}+d_{2}-2+\frac{1}{p_{1}+l_{2} T^{-1}+0.2} .
$$

Thus again the relevant measure in case (c) does not exceed $|I| / 32$.

CASE 4:

$$
l_{2} T^{-1}+p_{1}<7 / 4 \text {. }
$$

Let us estimate the expression $l_{2} T^{-1}+p_{1}$ from below. To do this we have to prove that $\left|P^{\prime}(x)\right| \asymp 2^{t\left(1-p_{1}\right)}$. By Taylor's formula for $P^{\prime}(x)$ at $\alpha$, we get

$$
P^{\prime}(x)=\sum_{j=0}^{n-1} \frac{P^{(j+1)}(\alpha)(x-\alpha)^{j}}{j !} .
$$

Clearly $\left|P^{\prime}(\alpha)\right| \asymp 2^{t\left(1-p_{1}\right)}$. The remaining terms of the sum satisfy

$$
\left|P^{(i)}(\alpha)(x-\alpha)^{i-1}\right| \ll 2^{t\left(1-p_{1}\right)}, \quad 2 \leq i \leq n .
$$

Since $\left|P^{\prime}(x)\right| \ll 2^{t / 3}$, we have $1-p_{1} \leq 1 / 3$ or equivalently $2 / 3 \leq p_{1}$. Thus we need to consider the system

$$
\left\{\begin{array}{l}
|P(x)|<2^{t(-n+v)} \\
\left|P^{\prime}(x)\right|<2^{t / 3} \\
2 / 3<l_{2} T^{-1}+p_{1}<7 / 4
\end{array}\right.
$$

All solutions of (37) with $\alpha_{1}$ being the closest root to $x$ are contained in the interval

$$
\sigma(P)=\left\{x \in I:\left|x-\alpha_{1}\right|<2^{t(-n+v)}\left|P^{\prime}\left(\alpha_{1}\right)\right|^{-1}\right\} .
$$

Apart from $\sigma(P)$ we also consider the following interval $\sigma_{1}(P)$, which contains $\sigma(P)$ :

$$
\sigma_{1}(P)=\left\{x \in I:\left|x-\alpha_{1}\right|<2^{t(v-0.9)}\left|P^{\prime}\left(\alpha_{1}\right)\right|^{-1}\right\} .
$$

From (38) and (39) we get

$$
\mu \sigma(P) \ll 2^{t(-n+v+1-v)} \mu \sigma_{1}(P)=2^{t(-n+0.9)} \mu \sigma_{1}(P) .
$$

Divide all polynomials in $\mathcal{P}_{n}$ into classes $\mathcal{P}_{\bar{b}}$ according to the $n-1$ first coefficients $\bar{b}=\left(a_{n}, a_{n-1}, \ldots, a_{2}\right)$. Obviously $\# \bar{b} \asymp 2^{t(n-1)}$.

(a) If $\mu \sigma_{1}\left(P_{1}\right) \cap \mu \sigma_{1}\left(P_{2}\right)<\frac{1}{2} \mu \sigma_{1}\left(P_{1}\right)$ then $\sum_{P \in \mathcal{P}_{\bar{b}}} \mu \sigma_{1}(P) \ll|I|$. Summing over all classes we obtain

$$
\sum_{\bar{b}} \sum_{P \in \mathcal{P}_{\bar{b}}} \mu \sigma(P) \leq 2^{n} 2^{t(n-1)} n 2^{t(-n+0.9)} 2|I| \leq n 2^{n+1} 2^{-0.1 t}|I| .
$$

(b) If $\mu \sigma_{1}\left(P_{1}\right) \cap \mu \sigma_{1}\left(P_{2}\right) \geq \frac{1}{2} \mu \sigma_{1}\left(P_{1}\right)$ we denote $R(x)=P_{1}(x)-P_{2}(x)$. Since $P_{1}$ and $P_{2}$ belong to the same class $\mathcal{P}_{\bar{b}}, R(x)$ is of the type $a x+b$. 
Moreover, taking into account the estimates of the polynomials and their derivatives we obtain

so

$$
\left\{\begin{array}{l}
|a x+b| \ll 2^{t(-0.9+v)}, \\
|a| \ll 2^{t\left(1-p_{1}\right)}
\end{array}\right.
$$

$$
\left|x+\frac{b}{a}\right| \ll 2^{t(-0.9+v)}|a|^{-1} .
$$

It is clear that inequality (40) holds for the whole essential interval. Summing estimates (40) first over all $b$ which do not exceed $c(n)|a||I|$, and then over all $a$, we obtain $c(n) 2^{t\left(-0.9+v+1-p_{1}\right)}|I|=c(n) 2^{t\left(v-p_{1}+0.1\right)}|I| \ll$ $2^{-0.1 t}|I|$. Let us sum the estimates of cases (a) and (b) over all $t>t_{0}$. We deduce that in Case 4 the measure of the set of those $x$ that satisfy (23) does not exceed $|I| / 32$. Altogether for Cases 1-4 the measure of the set $\mathcal{L}_{n}^{\prime \prime}(v)$ does not exceed $|I| / 4$, thus proving the theorem.

Acknowledgments. The authors are grateful to the referee and to the editorial team for their useful comments and to V. V. Beresnevich for his valuable help.

\section{References}

[1] V. Bernik, The metric theorem on the simultaneous approximation of zero by values of integral polynomials, Izv. Akad. Nauk SSSR Ser. Mat. 44 (1980), 24-45 (in Russian).

[2] -, On the exact order of approximation of zero by values of integral polynomials, Acta Arith. 53 (1989), 17-28 (in Russian).

[3] V. I. Bernik and M. M. Dodson, Metric Diophantine Approximation on Manifolds, Cambridge Tracts in Math. 137, Cambridge Univ. Press, 1999.

[4] K. Györy, Polynomials and binary forms with given discriminant, Publ. Math. Debrecen 69 (2006), 473-499.

[5] S. A. Katre and S. A. Khule, A discriminant criterion for matrices over orders in algebraic numbers fields to be sums of squares, in: Proc. Sympos. Algebra and Number Theory (Cochin, 1990), Publ. 20, Centre Math. Sci., Trivandrum, 1990, 31-38.

[6] -, -, Matrices over orders in algebraic number fields as sums of $k$-th powers, Proc. Amer. Math. Soc. 128 (2000), 671-675.

[7] V. G. Sprindžuk, Mahler's Problem in Metric Number Theory, Nauka i Tekhnika, Minsk, 1967 (in Russian); English transl.: Transl. Math. Monogr. 25, Amer. Math. Soc., Providenca, RI, 1969.

Institute of Mathematics

Surganova St. 11

220072, Minsk, Belarus

E-mail: bernik@im.bas-net.by olga_kukso@tut.by
Universität Bielefeld

Fakultät für Mathematik

Postfach 100131

33501 Bielefeld, Germany

E-mail: goetze@math.uni-bielefeld.de 